\title{
Measurement and Analysis of the Korean NDGPS Radiation Spectrum
}

\author{
Young-Wan Kim* and Suk-Kun Jee, Member, KIICE \\ Department of Radio Communication Engineering, Kunsan National University, Kunsan 573-701, Korea
}

\begin{abstract}
The Korean nationwide differential global positioning system (NDGPS) reference station transmits a global positioning system (GPS) enhancement signal using minimum shift keying modulation with a 200 bps data rate. The ocean-based DGPS covers the service area of $100 \mathrm{NM}$ with $300 \mathrm{~W}$ output power; on the other hand, the land-based DGPS transmits the output power of $500 \mathrm{~W}$, which covers the service area of $100 \mathrm{~km}$. The DGPS reference stations with high output power can radiate spurious signals, which may act as interference sources affecting the other DGPS reference stations or the wireless ground stations that utilize the medium frequency band. In this paper, the radiation spectrums of the DGPS reference stations are measured and analyzed in the spurious domain. The DGPS radiation spectrums are evaluated from the perspective of the interference effect.
\end{abstract}

Index Terms: DGPS, Radiation spectrum, NDGPS, DGPS receiver

\section{INTRODUCTION}

The Korean nationwide differential global positioning system (DGPS) consists of ocean-based DGPS for ocean service and land-based DGPS for land service. The oceanbased DGPS of 11 reference stations and the land-based DGPS of 6 reference stations are operating and providing differential GPS service nationwide [1]. The DGPS transmits an enhancement GPS signal that the receiver stations may use to correct their pseudo-ranges. The DGPS reference stations provide the service information using the minimum shift keying (MSK) modulation with a 200 bps data rate in the frequency range of $283.5-325 \mathrm{kHz}$ [2, 3].

The ocean-based DGPS covers the service area of 100 NM by the output power of $300 \mathrm{~W}$; on the other hand, the land-based DGPS transmits the output power of $500 \mathrm{~W}$, which covers the service area of $100 \mathrm{~km}$. The DGPS reference stations with high output power can radiate spurious signals, which may act as interference sources affecting the other DGPS reference stations or the wireless ground stations that utilize the medium frequency (MF) band. Also, the power amplifier (PA) of the DGPS system is operated in the switching mode; therefore, spurious signals are generated from the PA and may be radiated from a DGPS reference station. It is necessary to analyze the interference effect on other DGPS reference stations and user terminals from the spurious signals.

In this paper, the radiation spectrum characteristics of the DGPS reference station are measured and analyzed from the perspective of spectrum purity and the interference effect from spurious signals of the DGPS reference station. The output spectrums of the ocean-based DGPS of 11 reference stations and the land-based DGPS of 6 reference stations are measured near the DGPS system.

\section{DGPS REFERENCE STATION}

Fig. 1 shows the service areas of the Korean NDGPS that are provided from the ocean-based DGPS of 11 reference

Received 07 March 2012, Revised 13 April 2012, Accepted 20 April 2012

*Corresponding Author E-mail: ywkim@kunsan.ac.kr 
stations and land-based DGPS of 6 reference stations [1]. The ocean-based DGPS provides DGPS service for the ocean area, while the land-based DGPS covers the landbased service area that cannot be provided the service from the ocean-based DGPS. The NDGPS reference stations are operated with an output power of $500 \mathrm{~W}$ for the land-based DGPS and $300 \mathrm{~W}$ for the ocean-based DGPS. Therefore, the DGPS service areas overlap each other, which improve the service availability. On the other hand, the overlapped service area can cause interference with user terminals by spurious signals from another DGPS reference station.

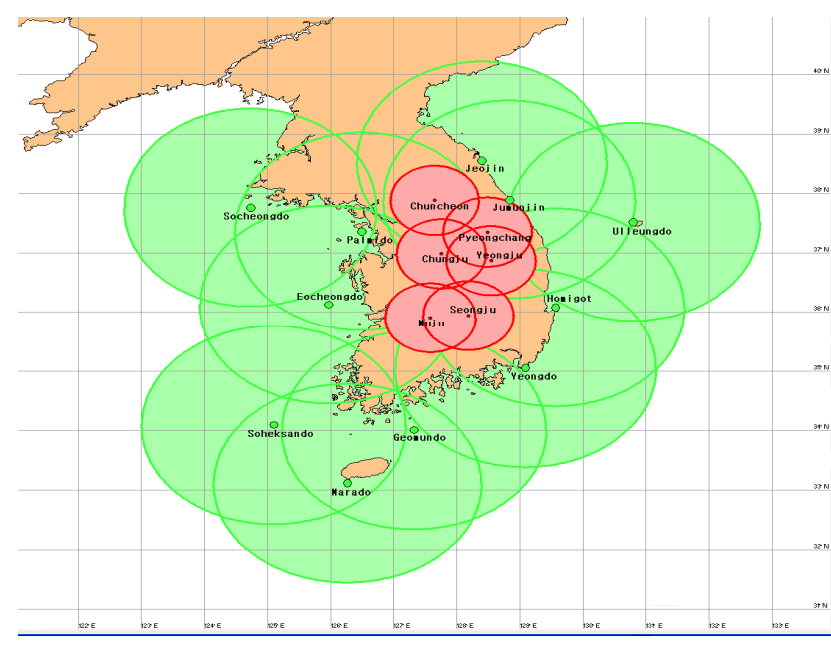

Fig. 1. Service areas of Korean nationwide differential global positioning system.

The spurious signals originate from the DGPS reference system, especially the power amplifier of the DGPS reference system. The DGPS reference system consists of an MSK modulator, power amplifier, and other units shown in Fig. 2.

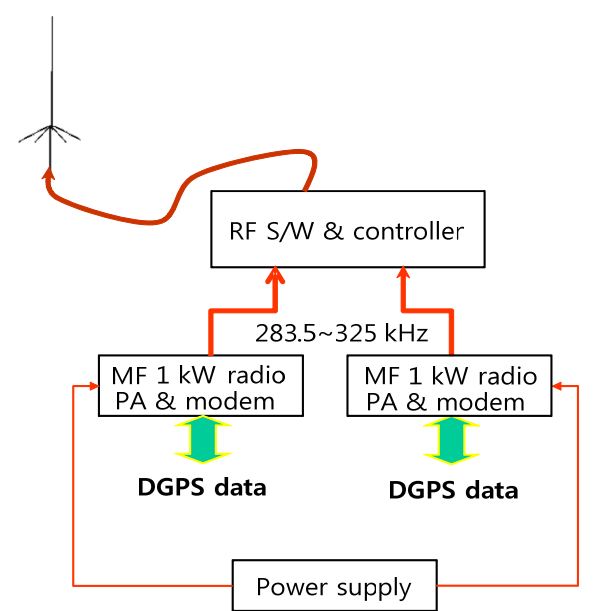

Fig. 2. Block diagram of differential global positioning system (DGPS) reference system. MF: medium frequency, PA: power amplifier, RF: radio frequency.
The MF power amplifier in the DGPS reference system is operated in the switching mode. In the switching operation mode, the harmonic signals besides a fundamental frequency signal are generated as shown in Fig. 3.

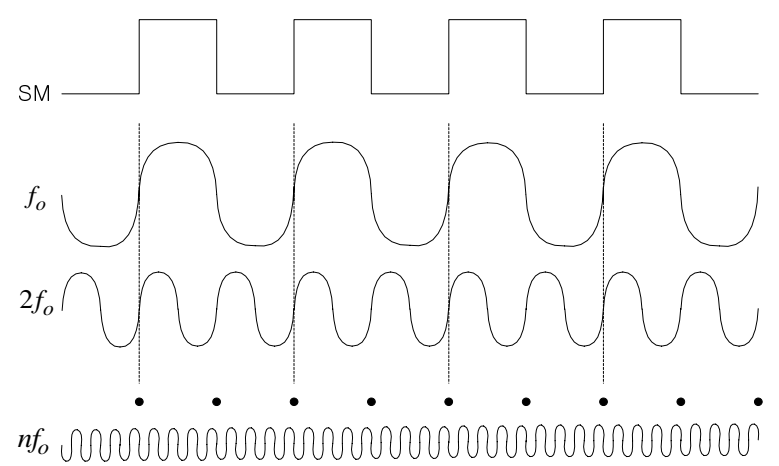

(a)

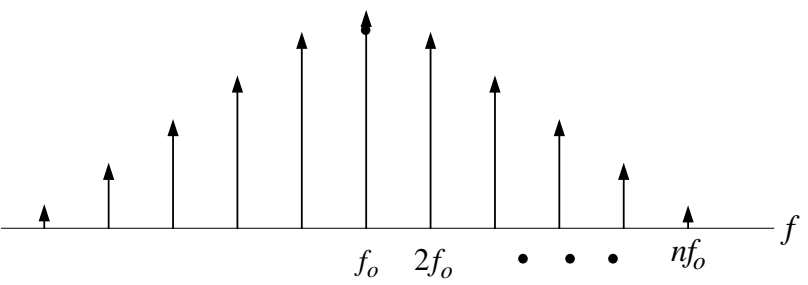

(b)

Fig. 3. Output signals of a power amplifier in the time domain (a) and frequency domain (b)

To reject harmonic signals, which can act as sources of interference, a band-pass filter is used in the output stage of the power amplifier. Furthermore, to eliminate the possibility of interference from another DGPS signal, the transmission frequencies of DGPS are allocated with a sufficient frequency offset. Table 1 shows transmission frequency allocation for the Korean DGPS. The DGPS transmission frequencies are allocated in the frequency range of $283.5 \mathrm{kHz}$ to $325 \mathrm{kHz}$ with a frequency offset of over $1 \mathrm{kHz}$ [1]. However, the antenna of the DGPS reference station is usually the top-loaded antenna type [4, 5]. The ground side of the MF antenna is a series of radials. Radials are crucial in the design of a top loaded antenna as the electrical properties of the antenna require the reaction between the top loaded portion and the ground system. The radiation pattern and input impedance of the DGPS has effects due to the ground plane [6]. The spurious signals of the DGPS may be affected by the antenna operating conditions and environment.

\section{SPECTRUM MEASUREMENT OF DGPS REFERENCE STATION}

To analyze the interference effect on other DGPS user terminals and other wireless ground stations due to the 
spurious signals, the radiation spectra of DGPS reference stations are measured and analyzed in the interference domain.

The radiation spectra are measured near the DGPS reference system and over two kilometers' distance from the DGPS reference station.

The radiation spectra near the DGPS reference system are typically measured as shown in Fig. 3b. The radiation spectrum near the DGPS reference system is shown in Fig. 4. The fundamental frequency, which is the desired radiation signal, and the harmonic signals are measured around the DGPS reference system. Far away from the DGPS reference station, however, the fundamental signal is only measured at a distance. Fig. 5 shows the fundamental spectrum measured from a distance.

Table 1. Transmission frequency of the Korean NDGPS reference station

\begin{tabular}{ccccc}
\hline Ref. station & Sochengdo & Palmido & Chungju & Echengdo \\
\hline $\begin{array}{c}\text { Frequency } \\
\text { (kHz) }\end{array}$ & 323 & 312 & 318 & 295 \\
\hline Modulation & \multicolumn{4}{c}{ MSK with 200 bps data rate } \\
\hline \hline Ref. station & Gemundo & Youngdo & Homigok & Ulleungdo \\
\hline $\begin{array}{c}\text { Frequency } \\
\text { (kHz) }\end{array}$ & 287 & 300 & 310 & 319 \\
\hline Modulation & \multicolumn{5}{c}{ MSK with 200 bps data rate } \\
\hline Ref. station & Pyeongchang & Youngju & Muju & Sengju \\
\hline $\begin{array}{c}\text { Frequency } \\
\text { (kHz) }\end{array}$ & 303 & 289 & 322 & 296 \\
\hline Modulation & \multicolumn{5}{c}{ MSK with 200 bps data rate } \\
\hline \hline Ref. station & Sohueksando & Marado & Jumunjin & Jeojin \\
\hline $\begin{array}{c}\text { Frequency } \\
\text { (kHz) }\end{array}$ & 298 & 290 & 295 & 292 \\
\hline Modulation & \multicolumn{5}{c}{ MSK with 200 bps data rate } \\
\hline
\end{tabular}

NDGPS: nationwide differential global positioning system, MSK: minimum shift keying.

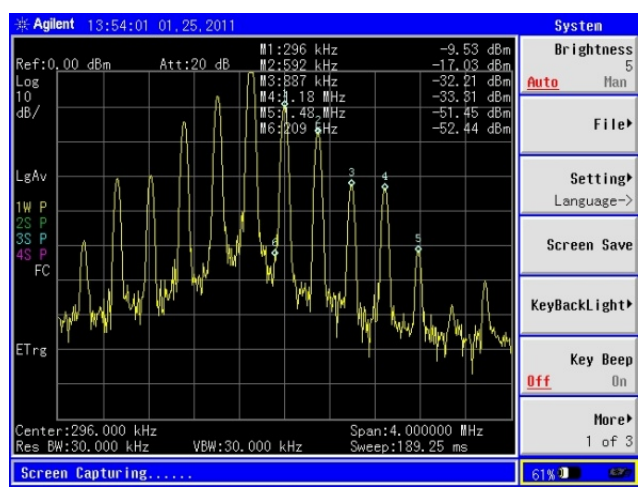

Fig. 4. Measured spectrum near the differential global positioning system reference system (Sengju).

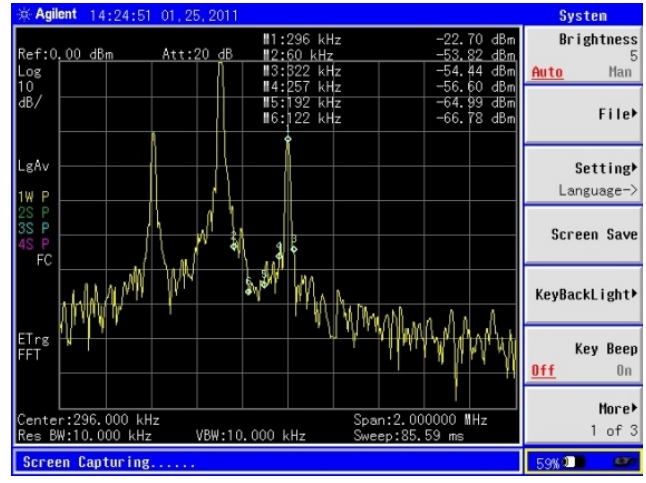

Fig. 5. Measured spectrum at $2 \mathrm{~km}$ distance from the differential global positioning system reference station (Sengju).

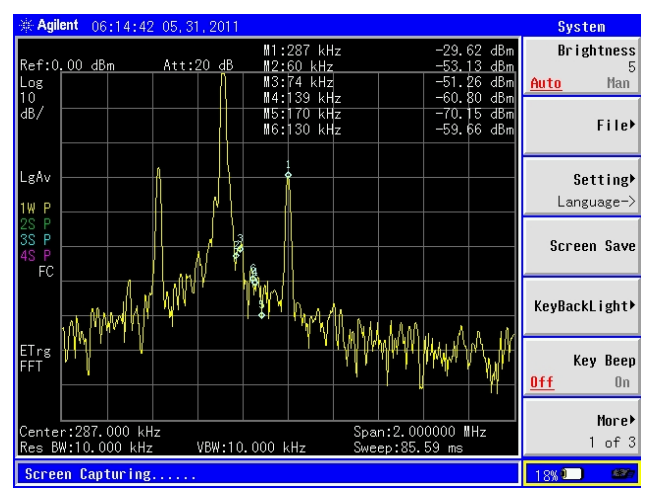

Fig. 6. Measured spectrum at the faraway distance from ocean-based differential global positioning system reference station (Gemundo).

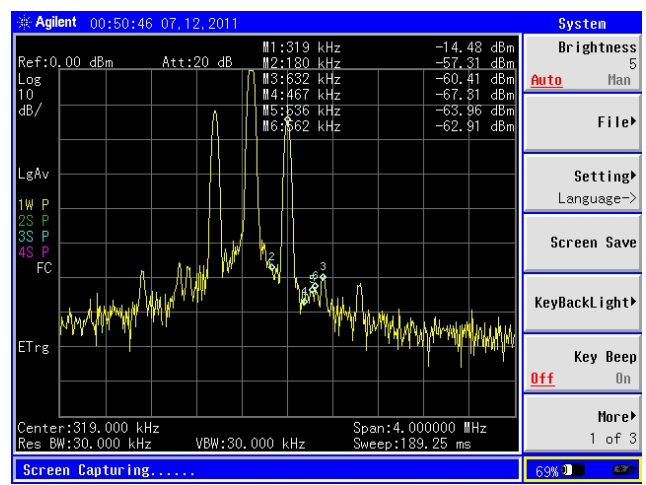

Fig. 7. Measured fundamental spectrum with second harmonic signals (Ulleungdo).

In the case of the ocean-based DGPS, the fundamental signal is measured far away from the DGPS reference station. The typical radiation spectrum for the ocean-based DGPS reference system is shown in Fig. 6.

The measured spectra are categorized into four patterns, a fundamental spectrum, a fundamental spectrum with 2nd harmonic signals, a fundamental spectrum with a spurious 
signal around the second harmonic frequency range, and a fundamental spectrum with spurious signals near the fundamental signal. Those spectra are shown in Figs. 6-9.

In the operation of the DGPS reference system, a large number of harmonic signals appear in the output signal domain (Fig. 4). The fundamental spectrum is only measured at a faraway distance (Figs. 5 and 6). In this case, the spurious signals besides the fundamental signal, which are evanescent signals do not propagate to a faraway distance. However, the second harmonic signal of DGPS was measured in the Ulleungdo DGPS reference station as shown in Fig. 7.

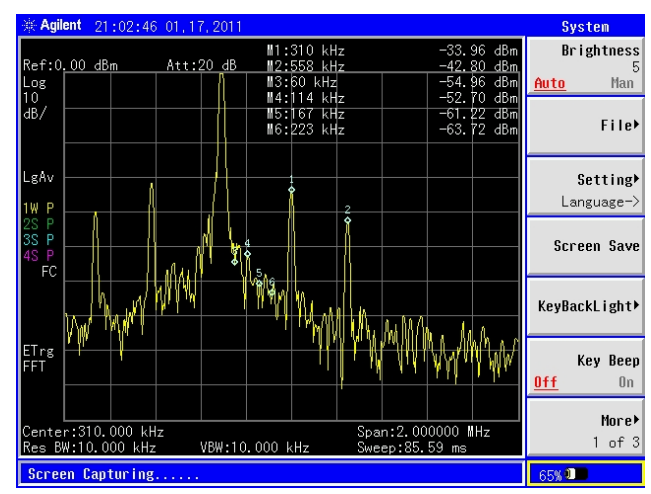

Fig. 8. Measured fundamental spectrum with spurious signal around the second harmonic frequency range (Homigok).

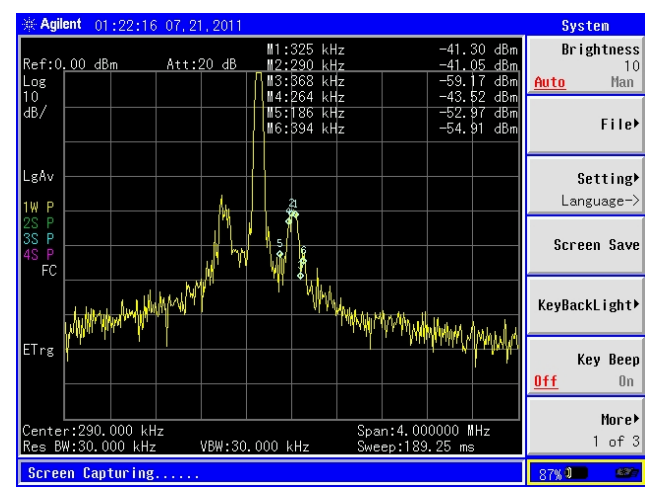

Fig. 9. Measured fundamental spectrum with spurious signals near the fundamental signal (Marado).

Fig. 10 shows the measured spectrum near the Ulleungdo DGPS reference system. Like the other DGPS reference systems, a number of harmonic signals including a fundamental signal appear around the DGPS reference system. As the propagation distance is increased, it is known from Fig. 10, and Fig. 7 that the second harmonic signal power decays from -20.56 to $-60.41 \mathrm{dBm}$ with about 39.9 $\mathrm{dB}$ propagation loss, while the fundamental signal power experiences a propagation loss of about $5.6 \mathrm{~dB}$ (from -8.93 to $-14.48 \mathrm{dBm})$. Although the second harmonic signal subsists within close range of the DGPS reference station, it is known that the harmonic signals do not radiate to a faraway distance via the DGPS antenna.

The fundamental spectrum with a spurious signal around the second harmonic frequency range was measured as in Fig. 8. In Fig. 8, the spurious signal is a signal with a frequency of $558 \mathrm{kHz}$, which bears no relation to the DGPS signal $(310 \mathrm{kHz})$ of the Homigok DGPS reference system. The signal may be a transmission signal of another wireless ground station using an MF frequency range. Spurious signals similar to Fig. 8 were measured for an ocean-based DGPS reference station (Echeongdo station with spurious signal of $547 \mathrm{kHz}$; Fig. 11). This is because the other MF wireless ground stations for ocean service are operating near a DGPS reference station.

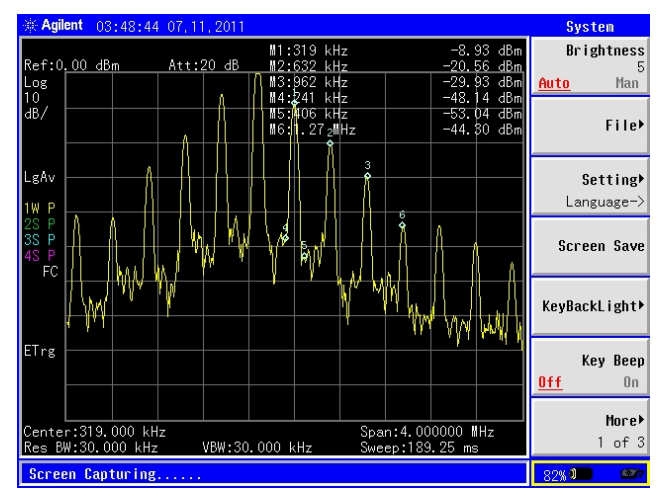

Fig. 10. Measured spectrum near the differential global positioning system reference system (Ulleungdo).

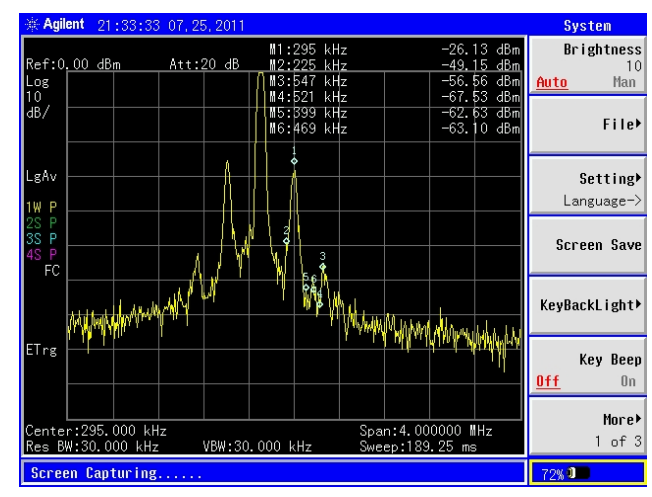

Fig. 11. Measured spectrum near a DGPS reference system (Echeongdo: $295 \mathrm{kHz}$ ).

Fig. 9 shows a fundamental spectrum with spurious signals near the fundamental signal. A large number of spurious signals near the fundamental signal were measured in the case of the Marado DGPS reference station. To analyze the effect of in-band transmission noise, the spurious signals close to the fundamental signal were evaluated in the frequency domain as shown in Fig. 12. It is known from Fig. 
12 that the in-band noise within a channel bandwidth of 500 $\mathrm{Hz}$ does not affect the DGPS reception performance. The spurious signals close to the fundamental signal are generated by other wireless ground stations and adjacent DGPS reference stations, which does not affect DGPS user terminals. This is because the DGPS receiver rejects the outof-band signals with a $40 \mathrm{~dB}$ rejection ratio.

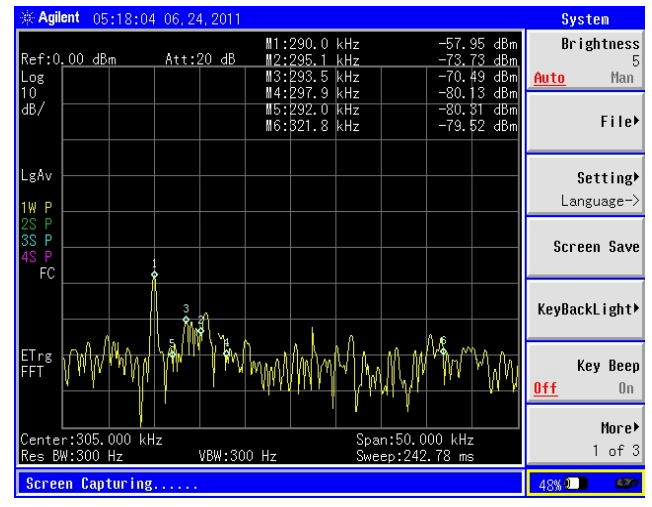

Fig. 12. Measured narrow-band spectrum for Marado reference station $(290 \mathrm{kHz})$.

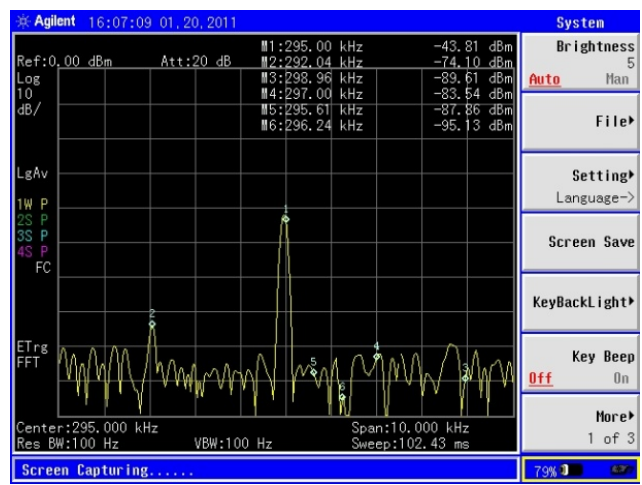

(a)

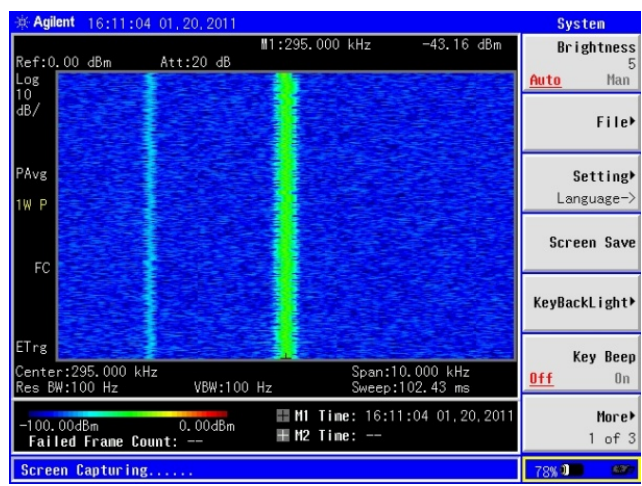

(b)

Fig. 13. Measured DGPS signals with adjacent DGPS channel (Jeojin, $292 \mathrm{kHz}$ and Jumunjin, $295 \mathrm{kHz}$.
There are adjacent DGPS signals around the radiation spectrum of the Marado reference station as shown in Fig. 12. The radiation signals of Echeongdo DGPS reference station $(295 \mathrm{kHz})$ and Sohueksando DGPS reference station (298 kHz) were measured as adjacent DGPS channels.

The MF DGPS signal that propagates as a surface wave has effects due to the conductivity of the propagation media. In a propagation plane with a good conductivity, the DGPS signal propagates to a faraway distance. The ocean-based DGPS for ocean service, which have better conductivity than that of the ground plane, can service a wider area than can the land-based DGPS. Therefore, a number of DGPS signals can be propagated in a service area. Fig. 13 shows a DGPS spectrum with a propagation signal of an adjacent DGPS reference station, where the channel frequencies of Jeojin (adjacent channel) and Jumunjin reference station are $293 \mathrm{kHz}$ and $295 \mathrm{kHz}$, respectively.

\section{CONCLUSIONS}

The radiation spectra of Korean DGPS reference stations using the MF band are measured and analyzed from the perspective of the interference effects due to spurious signals. The spurious signals disappear at a faraway distance, while a number of the spurious signals are measured near the DGPS reference station. The desired fundamental signal of the DGPS reference station is only radiated via the MF transmitting antenna.

The harmonic signals of the DGPS signal originate from the power amplifier of the DGPS system. The signals does not radiate via the MF DGPS antenna. Though the spurious signals close to the fundamental signal are generated by other wireless ground stations and adjacent DGPS reference stations, it is known that the spurious signals do not affect the DGPS user terminals.

\section{REFERENCES}

[1] Ministry of Land, Transport and Maritime Affairs (Korea). DGPS Centeral Office [Internet]. Available: http://www.ndgps.go.kr/.

[2] Korea Aerospace Research Institute, "Study on the development of DGPS receiver,” Ministry of Land, Transport and Maritime Affairs, Gwacheon, Final report, 2003.

[3] G. S. Yang, "The measurements on a field strength and SNR for national wide differential global positioning systems in Korea," dissertation, Chungju University, Chungju, 2010.

[4] V. Trainotti and L. A. Dorado, "Short low- and medium-frequency antenna performance,” IEEE Antennas and Propagation Magazine, vol. 47, no. 5, pp. 66-90, 2005.

[5] V. Trainotti and L. A. Dorado, "On the crossed field antenna performance,” IEEE Transactions on Broadcasting, vol. 52, no. 3, pp. 299-317, 2006.

[6] Y. W. Kim, "Propagation analysis of DGPS antenna for radial ground and obstacle”, International Journal of Information and Communication Engineering, vol. 9, no. 4, pp. 363-368, 2011. 


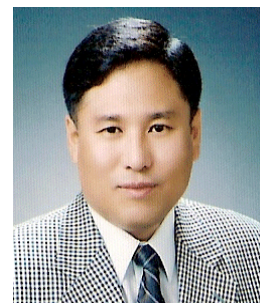

\section{Young Wan Kim}

received the M.S. and Ph.D. degrees in electronic engineering from Kyung-Pook National University, Taegu, and from Chung Nam National University, Daejeon, Korea in 1985 and 2003, respectively. From 1985 to 1992 , he was with the OPC Research Center, Korea, where he was involved in the development of the TVRO and the TVR system. From 1992 to 2004, he was with the Radio and Broadcasting Technology Lab, in the Electronics and Telecommunications Research Institute (ETRI) in Taejon, Korea. Since 2004, he has been with Kunsan National University and is presently a Professor in the Department of Radio Communication Engineering. His research interests are in digital satellite communication, TT\&C systems for satellites, digital broadcasting systems, radio communication technologies, and microwave devices.

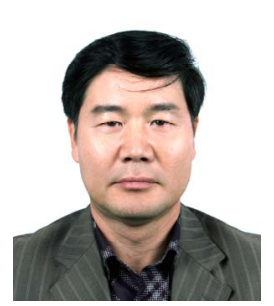

\section{Suk Kun Jee}

received the M.S. and Ph.D. degrees in electronic engineering from Pu-Kyong National University, Busan, Korea in 1990 and 1997, respectively. Since 1990, he has been with Kunsan National University and is presently a Professor in the Department of Radio Communication Engineering. His research interests are in digital signal processing, active control systems. 